\title{
ABTRAGUNGS- UND VERWITTERUNGSFORMEN IM LIGURISCH-EMILIANISCHEN APENNIN*
}

\author{
RUDOLF ULLMANN
}

Der ligurisch-emilianische Apennin gehört zu jenen Gebirgen des Mittelmeergebietes, deren Relief, gemessen an geologischen Zeiträumen, in rascher und stetiger Umformung begriffen ist. Während der Hauptregenperiode, im Herbst und Frühjahr, werden fast täglich - für den Beobachter deutlich sichtbar - morphologische V'orgänge ausgelöst, die scharf umrissene, meist kurzlebige Formen schaffen, alte verwischen oder ganz auslöschen. Die Auswirkungen dieser als «Frane» bezeichneten Erscheinungen auf die Siedlungen, die Agrarwirtschaft und die Verkehrswege stehen vielfach denen der Erdbeben kaum nach.

Von deutscher Seite aus machte erstmals Th. Fischer (1893) auf diese verheerenden Vorgänge aufmerksam. Einige Jahre später untersuchte G. Braun (19o7) die Frane und Calanche sowie die tief eingeschnittenen Quellkessel im Apennin südlich Modena-Bologna und im Volterrano. Er konnte nachweisen, da $B$ die Frane bei der Gestaltung der Täler in entscheidendem Maße beteiligt sind. 1929 veröffentlichte $H$. Kanter einen Aufsatz über junge Abtragungserscheinungen im Osten Kalabriens und der südlichen Basilicata. F. Tichy griff 1957 das «Franeproblem» im $\mathrm{Zu}$ sammenhang mit den Folgen der Entwaldung in der Basilicata wieder auf und verglich aufgrund von mehrjährigen Beobachtungen das Ausma $B$ der morphologischen Veränderungen. Von den zahlreichen italienischen Arbeiten - mehr als 100 Titel - , die sich mit den «Franeerscheinungen» befassen und in den verschiedensten $Z$ eitschriften veröffentlicht sind, mögen nur einige wichtige genannt werden. Schon 1889 beschrieb G. Trabucco die «Frane» und ihre zerstörenden Wirkungen im Piacentino. R. Almagia (1907/10) dehnte die «Franeuntersuchungen» auf die gesamte $\mathrm{Halb}$ insel aus und legte für diese besonderen morphologischen Erscheinungen die italienischen Termini fest1. B. Castiglioni untersuchte 1935 den Formenschatz im Pliozän des Mittelapennins. In

Neben diesen erwähnten Abtragungsformen und -vorgängen treten in der Hochregion und in den tieferen Lagen des ligurisch-emilianischen Apennins Blockmeere, Block- und Schutthalden, Felsburgen und besondere Verwitterungsformen in Erscheinung, die bislang in der Literatur unberücksichtigt geblieben sind. Im vorliegenden Aufsatz soll daher in einem regional begrenzten Gebiet die vorhandene Vielfalt der Formen aufgezeigt werden. Zugleich wird versucht, die Vorgänge sowie die Ursachenreihen zu klären, die zur Bildung dieser Formen führen. Das Hauptaugenmerk wird auf die Frage gerichtet sein, welche geologisch-physischgeographischen und anthropogenen Einflüsse für die ständige und relativ schnelle Umwandlung des Reliefs und damit für die Entstehung und das Aussehen der einzelnen Formen und Vorgänge verantwortlich gemacht werden müssen. Aus dieser Fragestellung muß sich ergeben, ob bestimmte Formen und Vorgänge nur eine Folge der heutigen klimatischen Verhältnisse oder der menschlichen Einwirkungen sind. - Abgesehen von einer Diskussion der Frage der pleistozänen Schneegrenze wird der eiszeitliche Formenschatz von der Untersuchung ausgeschlossen. - Um keine terminologischen Unklarheiten aufkommen zu lassen, werden als Frane nur die Vorgänge der großen, langsamen Massenbewegungen sowie die aus ihnen resultierenden Formen - die in Mitteleuropa kein Analogon haben - bezeichnet. Somit braucht nicht auf die von verschiedenen Autoren vorgenommene Frane-Klassifikation (Heim 1882, Braun 1907, Almagia 19o7/1o, Maull 1958) und auf den neugeprägten Begriff «mediterrane Solifluktion» (Tichy 1959) eingegangen zu werden. Denn alle anderen in der Literatur als Frane geschilderten Vorgänge und Formen können ohne Schwierigkeiten nach der gebräuchlichen deutschen Nomenklatur, den Erd- oder Bergschlipfen, den Fels- oder Bergstürzen zugeordnet werden. Die übrigen morphologischen Erscheinungen werden gemäß den Begriffsbestimmungen von Wilhelmy (1958) und Verfasser (1959) definiert.

\section{Morphologisch-lithologischer Überblick}

Das Untersuchungsgebiet ist zwischen den Pässen Giovi $(472 \mathrm{~m})$ im W und Cisa (1039 m) im E gelegen und entspricht in seiner Ausdehnung mit ca. $8000 \mathrm{~km}^{2}$ ungefähr der 1,5-fachen Größe des Schwarzwaldes. In seinen Großformen unterscheidet es sich wesentlich von den anderen Be-

* synonym Nordapennin.

1 R. Almagia übernahm seine 4 Hauptfranetypen von A.Heim (1882).

neuerer Zeit beschäftigten sich F. Penta (1956/59) und E. Roveri (1960) mit dem Mechanismus der $\ll$ Frane». 
reichen des Apennins; westlich des Giovi schließt sich die niedrige Narbenzone von Sestri-Voltaggio an, während östlich des Cisa-Passes der eigentliche Kettenapennin einsetzt. Durch die hydrographischen Gegebenheiten ist die Hochregion in einzelne Gebirgsstöcke aufgelöst, die im W aus Kalkmergeln (Alberese) und im $\mathrm{E}$ aus Ophiolithen aufgebaut werden. Nur in der näheren Umgebung des höchsten Gipfels, des Mte Maggiorasca $(1803 \mathrm{~m})$, treten Hochgebirgsformen auf, die sowohl durch Härte der ophiolithischen Gesteine, als auch insbesondere durch die eiszeitliche Überformung zu erklären sind.

Die S-Abdachung zeigt breite, steilwandige Quelltrichter, die durch Absätze und Sporne gegliedert werden und sich seewärts zu tief eingesägten Tälern verengen. Angezapfte Täler, die in jüngster geologischer Vergangenheit noch zur Adria hin entwässerten, geben Zeugnis von dem bis heute nicht ruhenden Kampf um die Wasserscheide. An Gesteinen sind vorwiegend Kalkmergel und Kalkton (Alberese), die in der Nähe von Sestri-Voltaggio (bis ca. $12 \mathrm{~km}$ ) metamorphosiert sind, und Macigno vertreten.

Die N-Abdachung dagegen läßt typischen Mittelgebirgscharakter erkennen. Weiche oberkretazische Tone (Argille scagliose), tertiäre Mergel und Sandsteine mit dazwischen geschalteten Kalkbänken bauen die schmalen Bergrücken und Verebnungsflächen auf, die aber an ihren Rändern an keiner Stelle mehr intakt sind, sondern von den Tälern her durch Racheln (Calanche) zerschnitten werden.

Die Täler sind breitsohlig, Mittel- und Unterläufe hoch aufgeschottert. Verschiedentlich haben sich einige Terrassenreste ca. 5 bis $6 \mathrm{~m}$ über dem heutigen Schotterbett erhalten. Steilhänge sind nur im Macigno südlich Bobbio und im W zwischen Scrivia und Scuropasso anzutreffen, wo das Oligozän als hartes Konglomerat ausgebildet ist.

\section{Klimatische Übersicht}

Klimatisch zeichnet sich der nordwestliche Hochapennin durch bedeutende Niederschlagsmengen $(2800 \mathrm{~mm})^{2}$ aus und bildet zugleich eine wichtige Klimascheide. Gehört die schmale küstennahe Zone, die Riviera, zum mild-ausgeglichenen Mediterranklima, so trägt die im $\mathrm{N}$ des Apennins liegende Poebene ausgesprochen kontinentale Züge. Diese klimatischen Gegensätze wandeln sich entsprechend der Höhenlage auf der ligurischen und emilianischen Abdachung ab. Die Wintertemperaturen der Riviera, deren Saum mit der Höhengrenze der bis durchschnittlich $400 \mathrm{~m}$ hinaufsteigenden Olive zusammenfällt, sind gemäßigt. Je nach der Exposition der Hänge und Täler erreichen die mittleren Januartemperaturen 4 bis 8 Grad und sinken allmählich ab. Erst in $1500 \mathrm{~m}$ Höhe erfolgt eine rasche Abnahme auf -6 bis $-12 \mathrm{Grad}$. Auf der emilianischen Seite, in der Poebene, den Collini und auf den Höhen bis zu $1000 \mathrm{~m}$ liegen die Januarmittel zwischen -1 bis $-6 \mathrm{Grad}$. Im Sommer werden an der Küste Mitteltemperaturen von 23, in den Höhenlagen über $1500 \mathrm{~m}$ bis zu 18 und auf der N-Abdachung, zur Poebene hin, 3o Grad gemessen. Weniger ausgeprägt ist der unterschiedliche Charakter der N- und S-Seite in bezug auf Niederschlagsmenge und -verteilung. Fallen im Jahresmittel an der Riviera 1000 bis $1200 \mathrm{~mm}$, so wachsen die Werte mit zunehmender Höhe schnell an. Am Mte Maggiorasca sind es $2800 \mathrm{~mm}$ und die hochgelegenen Täler der Trebbia und des Taro erhalten durchschnittlich $2000 \mathrm{~mm}$ Niederschlag. Eine Verminderung erfolgt auf der N-Seite; die bis zu $1000 \mathrm{~m}$ aufragenden Gipfel erreichen Werte von $1100 \mathrm{~mm}$, die sich erst am äußersten Rand des Apennins stark verringern (Piacenza am Po $600 \mathrm{~mm})$.

Die Niederschläge verteilen sich im Norden wie im Süden des Gebirges im wesentlichen auf die Herbst- und Frühjahrsmonate. Oktober und November sind mit $38 \%$ der Jahresmenge am regenreichsten. Eine sommerliche Trockenzeit ist nur in der Poebene eingeschaltet. Im Gebirge selbst tritt eine eigentliche Niederschlagspause nicht ein.

\section{ERD- UND BERGSCHLIPFE3}

Die im ligurisch-emilianischen Apennin am häufigsten auftretenden Abtragungsvorgänge sind oberflächliche Rutschungen. Sie ähneln jenen aus den Opalinustonen und Keupermergeln der schwäbisch-fränkischen Schichtstufenlandschaft bekannten 10 bis $300 \mathrm{~m}^{2}$ großen und 30 bis $100 \mathrm{~cm}$ tiefen Erdschlipfen, die im Frühjahr Wiesen und Felder als braune oder graue Flecke - je nach der Gesteins- bzw. Bodenfarbe - durchsetzen. Auf Hängen bis zu 20 Grad Neigung rutscht das Lockermaterial meist flächenhaft, auf steileren fast immer streifenförmig ab. Abrißnische und Gleitbahnränder sind

2 Die Apuanischen Alpen mit etwas mehr als $3000 \mathrm{~mm}$ und die Hochregion des Untersuchungsgebietes mit $2800 \mathrm{~mm}$ Niederschlag sind die niederschlagsreichsten Gebiete der gesamten Halbinsel.

$3 \mathrm{Zu}$ den Beispielen vergl. Übersichtsskizze 1. 
nur für kurze Zeit deutlich ausgeprägt; schon der nächste Regenguß verwischt die Umrisse und spätestens im Herbst beim Pflügen wird das zu Wülsten von 40 bis $70 \mathrm{~cm}$ Höhe angehäufte Material eingeebnet. Was zurückbleibt, ist das unruhige Relief, das als charakteristisches Kennzeichen der Gebiete oberflächlicher Rutschungen gelten kann.

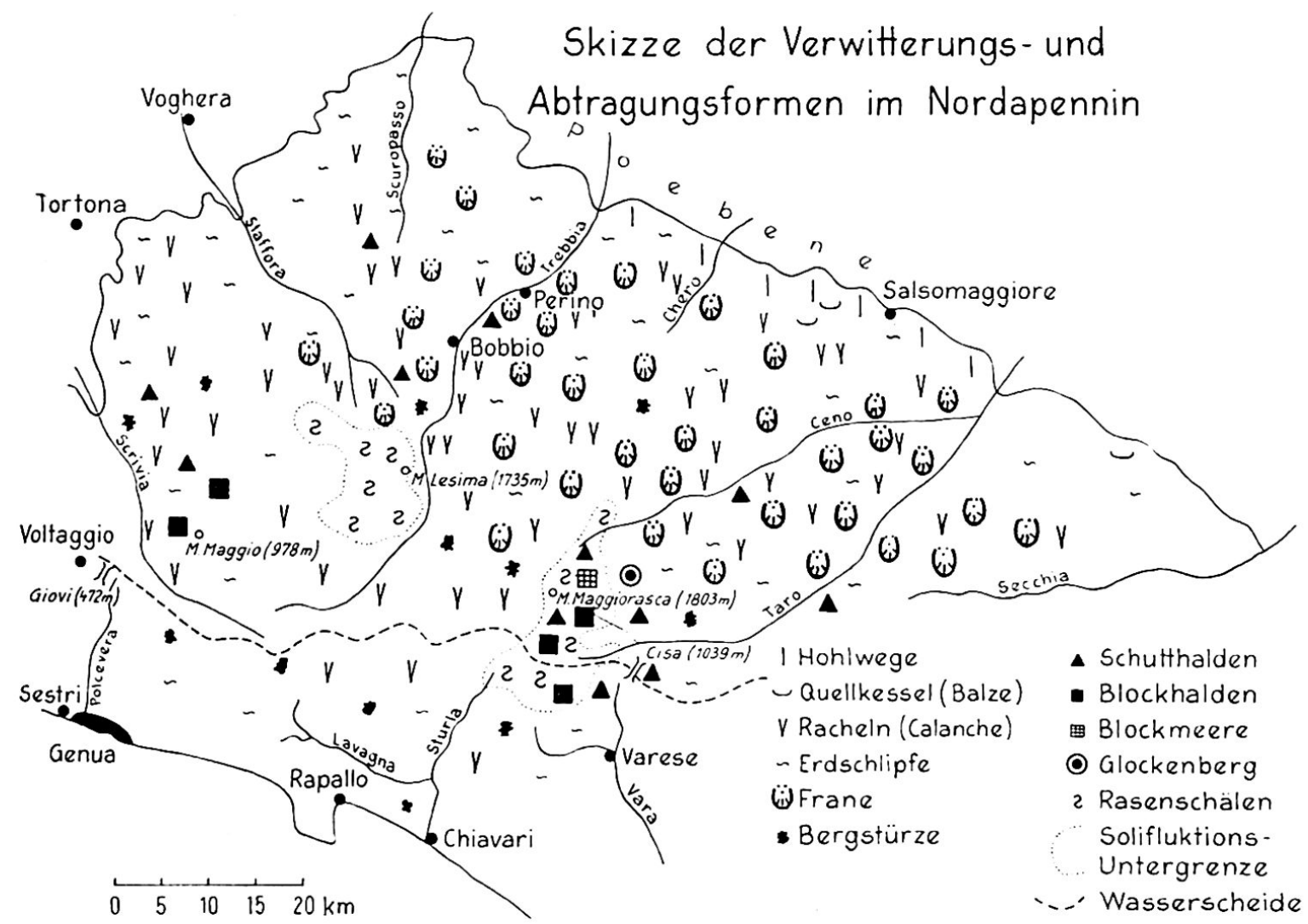

Abb. 1

Die Erdschlipfe kommen auf den mit einem tiefgründigen, lehmigen Boden (ferretto) überzogenen Riedeln beiderseits der nach NE zu geöffneten Täler vor, ebenso aber auch im Bereich der Mergel und Schuppentone, die fast überall auf der $\mathrm{N}$ - und S-Abdachung anstehen. Doch sind sie auf das offene Land beschränkt, wobei ihre obere Grenze bei $1200 \mathrm{~m}$ erreicht wird, die im Nordwestapennin zugleich der Siedlungsgrenze entspricht (Pei $1127 \mathrm{~m}$, Carta d'Italia 1:25 000, 71, II SO) ${ }^{4}$. Damit wird die Grundvoraussetzung für die Entstehung der Erdschlipfe berührt: die Entwaldung. Jedoch sind als allgemeine Bedingungen für das Auftreten der Erdschlipfe, lehmige Böden, anstehende Tone oder feinerdige Bestandteile im Hangschutt zu nennen, die große Mengen Wasser aufzunehmen vermögen. Handelt es sich beim Gesteinsuntergrund des Lockermaterials um wasserdurchläßige Kalk- oder Sandsteine, so muß die darüberliegende wasseraufnahmefähige Schicht mindestens $50 \mathrm{~cm}$ betragen, damit es zu Bewegungsvorgängen dieser Art kommen kann. Als auslösende Ursachen kommen die Herbst- und Frühjahrsniederschläge und in einzelnen Fällen auch die künstlich geschaffenen Wegeinschnitte in Frage.

\section{FRANE}

Als vergrößertes Abbild der Erdschlipfe, jedoch mit ganz anderen Auswirkungen, können die großen, langsamen Massenbewegungen, die Frane im eigentlichen Sinne, angesehen werden (Abb. 2).

4 Im folgenden Text werden nur die Blattnummern der Carta d'Italia 1:25 ooo angegeben. 


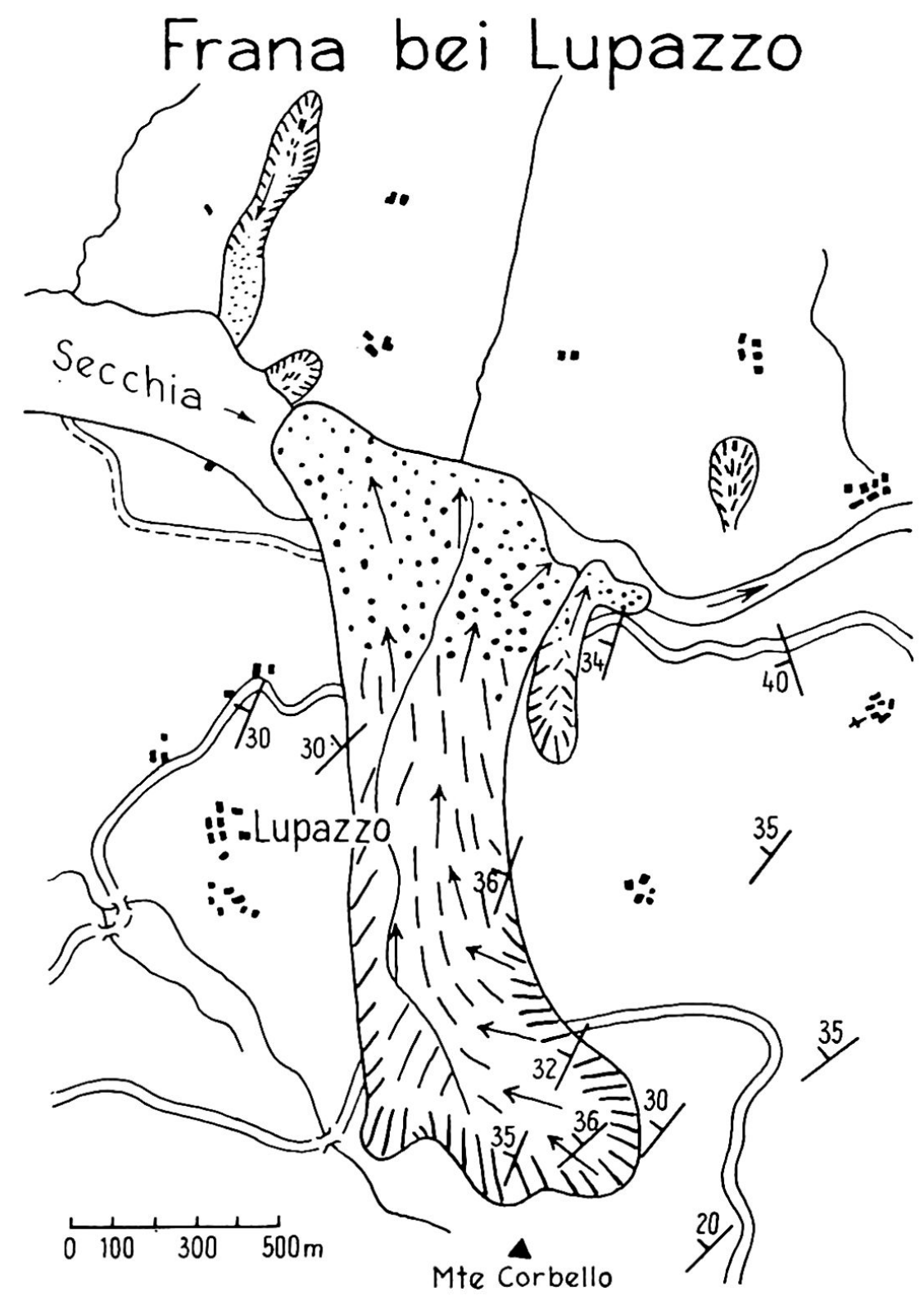

Abb. 2: Frana

Im Frühjahr 1960 kam es im mittleren Secchia-Tal bei Lupazzo (85, I SO) zur Bildung einer $2 \mathrm{~km}$ langen und $400 \mathrm{~m}$ breiten Frana, die eine $3000 \mathrm{~m}^{2}$ große Abrißnische am Mte Corbello (716 m) hinterließ. Im mittleren Abschnitt der Rutschung zeichneten sich scharf markierte Gleitbahnränder ab, während die abgeglittenen Erdund Gesteinsmassen das Secchia-Tal auf einer Breite von $600 \mathrm{~m}$ abdämmten, wodurch der Fluß auf ca. $8 \mathrm{~km}$ Länge aufgestaut wurde. Der Vorgang, der mehr als 2 Monate andauerte, zerstörte Felder, Reb- und Obstgärten und unterbrach die beiden Straßen zwischen Cerredolo und Toana. Wie aus dem Profil (Abb. 3) ersichtlich ist, wurde durch den in N-Richtung zur Secchia entwässernden Rio die ca. $30 \mathrm{~m}$ mächtige Kalkmergelschicht bis auf die tonige Unterlage zerschnitten und aus ihrem Zusammenhang gelöst. So begann das Gesteinspaket auf den mit 36 Grad nach NW einfallenden Schuppentonen abzugleiten und schwenkte beim Erreichen des Rio in dessen ehemalige Laufrichtung nach $\mathrm{N}$ um. Die mit Wasser vollgesogene tonige Unterlage gab eine vorzügliche Gleitbahn ab.

Unter ähnlichen geologischen Voraussetzungen erfolgte im Januar 1961 bei Perino im Trebbia-Tal (72, III NO) eine Frana, die 23 Häuser zerstörte und die Straße 


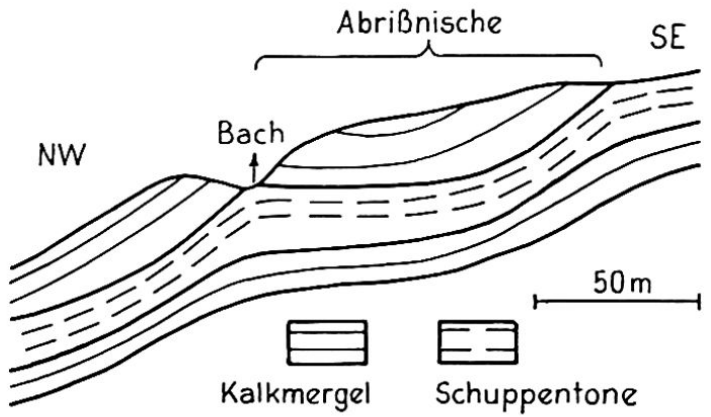

Abb. 3: Profll der Frana bei Lupazzo

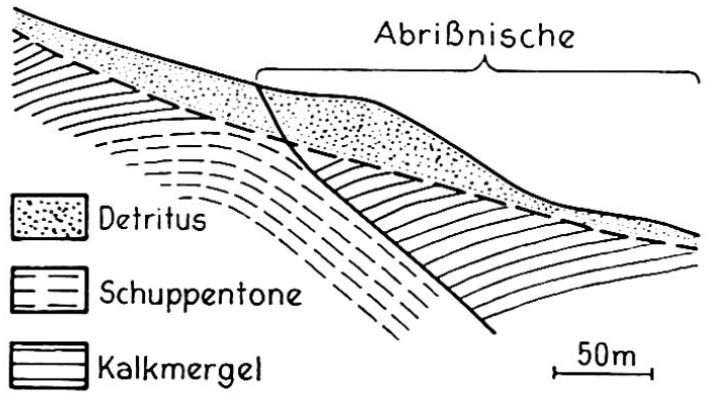

Abb. 4: Typische Lagerungsverhältnisse für die Entstehung von Franen

Genua-Piacenza ins Flußbett hinabriß. Wiederum bildeten Tone (scisti policromi) die Rutschfläche für die $20 \mathrm{~m}$ mächtigen Kalkmergel, die auf einer Länge von 1,5 km und $500 \mathrm{~m}$ Breite in Bewegung gerieten, aber im gesamten nur ca. $60 \mathrm{~m}$ weit abglitten. Eine Abrißnische bildete sich wegen der relativ geringen Abwärtsbewegung nicht aus, wie sich auch keine Gleitbahnränder abzeichneten, da die Frana durch zwei tief eingekerbte Bäche begrenzt wurde. Das ganze Abrißgebiet und der mittlere Abschnitt gliederten sich in einzelne Stufen auf, wobei im oberen Teil Absenkungsbeträge von 50 bis $80 \mathrm{~cm}$, im mittleren von 5 bis $10 \mathrm{~m}$ gemessen werden konnten. Im Ablagerungsgebiet bauten sich die abgeglittenen Gesteinsmassen gletscherzungenförmig ins Tal der Trebbia vor.

Der 5 Monate anhaltende Bewegungsvorgang begann wenige $m$ oberhalb der Talsohle der Trebbia, pflanzte sich hangaufwärts zu fort und erreichte in den ersten Tagen Geschwindigkeiten von 1,5 bis $2 \mathrm{~m}$, die sich im Juni auf 10 bis $15 \mathrm{~cm}$ reduzierten. Der Winkel der talwärts zu einfallenden Gesteinsschichten betrug 6 bis 8 Grad. Die beiden Beispiele ${ }^{5}$, die als typisch gelten dürfen, zeigen, daß die Form der Frane durch mehr oder minder gut ausgeprägte Abrißnischen und Gleitbahnränder sowie durch das gletscherzungenförmig abgelagerte Material bestimmt wird. Als besonderes Merkmal des Abtragungsvorganges ist das langsame Abgleiten anzusehen. Die Voraussetzung für die Entstehung der Frane bilden Charakter und Lagerungsverhältnisse der Gesteine. Es müssen wasserdurchlässige Schichten, deren Lagerung nur von untergeordneter Bedeutung ist, über wasserundurchlässigen, hangparellel einfallenden Tonen liegen, die die Gleitbahn abgeben (Abb. 4).

Je steiler der Einfallswinkel, um so schneller ist naturgemäß der Bewegungsablauf, der immer im unteren Abschnitt beginnt und sich nach oben zu fortsetzt. Doch ist für das langsame Abgleiten in erster Linie die überaus große Kohäsion der Tone verantwortlich zu machen. Hinzu kommt, daß das durch erosive Vorgänge aus dem Gesteinsverband herausgelöste Schichtpaket nicht in seiner Gesamtheit, sondern in einzelnen Stücken abrutscht. Die Schollen verkippen, sacken in sich zusammen, verteilen dadurch ihr Gewicht auf eine größere Reibungsfläche und können deshalb nur langsam abgleiten. Hieraus wird verständlich, daß die abgelagerten Erd- und Gesteinsmassen keinen Zusammenhang des ehemaligen Schichtverbandes mehr erkennen lassen, wie das für die in Mitteleuropa zuweilen auftretenden Bergrutsche charakteristisch ist. Sind die Lagerungsverhältnisse umgekehrt, d. h. bilden wasserundurchlässige Schichten die Oberfläche, so kommt es keineswegs zu Franeerscheinungen, sondern zu Erdschlipfen.

Die Verbreitung der Frane ist an die Alberese (Kalkmergel und Kalkton)- und Argille scagliose (Schuppentone)-Serien gebunden. Obwohl diese Gesteine im ganzen

5 Die Frana bei Lupazzo wäre nach der Nomenklatur von Almagia eine «Frana per scivolamento» = Gleitfrana, die bei Perino eine «Frana per cedimento»= Absinkfrana. Aus den Beschreibungen dürfte jedoch ersichtlich geworden sein, daß beide großen Massenbewegungen «Gleitfrane» sind; denn ein weiteres Abgleiten oder Absinken der Frana bei Perino ist nur durch die Trebbia verhindert worden. 
Untersuchungsgebiet anstehen, treten Frane fast ausnahmslos auf der emilianischen Abdachung bis zu 1100 m Höhe auf. Demzufolge kann für ihre Entstehung nicht ausschließlich die Gesteinsbeschaffenheit maßgebend sein.

Im Vergleich zur Hochregion und zur ligurischen Seite ist der emilianische Apennin viel weniger zerschnitten. Seine Hänge haben Neigungen zwischen 15 und 20 Grad. Verebnungsflächen und Rücken sind in vielen Bereichen zu erkennen. Hierfür sind als Gründe der asymmentrische Bau des Gebirges und die etwas geringeren Niederschläge auf der Leeseite anzuführen. Im Sommer wird die Bodendecke, auch wenn sie Niederwald (bosco cedui) trägt, durch Trockenrisse zerklüftet. Dem tonigen Untergrund wird kein Regenwasser zugeführt und er bleibt verhältnismäßig fest. Die Sommerniederschläge (Gewittergüsse) durchfeuchten größtenteils nur die Bodendecke und verlieren sich in den Klüften der mächtigen wasserdurchlässigen Schichten. Dagegen können in der Regenzeit die Niederschläge schnell zu den Tonschichten hinabgeführt werden. Diese saugen sich voll, erfahren eine Gewichtszunahme und deformieren sich zu einer aufgequollenen, zähflüssigen Masse, auf der die aus dem Gesteinsverband gleichsam herausgesägten, wasserdurchlässigen Schichtpakete in der oben erwähnten Weise abgleiten können. Hier, auf der relativ flachen emilianischen Abdachung mit ihren etwas geringeren Niederschlägen, ist das Hauptverbreitungsgebiet der Frane.

Auf der ligurischen Abdachung dagegen bewirkte von vornherein der Abbruch zum Ligurischen Meer ein steiles Relief. Die etwas größeren Niederschlagsmengen zerschnitten es intensiv. Jedoch ist der sommerliche Niederschlagsmangel dem der emilianischen Seite vergleichbar. Sind hier auch ähnliche Lagerungsverhältnisse der Gesteine anzutreffen (wie im Norden), so lassen doch die durch tektonische Vorgänge geschaffenen steilen Reliefverhältnisse keine langsamen Massenbewegungen zu. Was in Ligurien an großen Massenbewegungen gelegentlich zu beobachten ist, sind die weiter unten beschriebenen Bergstürze, die fast ausschließlich durch den Straßen- und Eisenbahnbau ausgelöst werden 6 .

- Daß es in Ligurien oftmals zu Terrasseneinstürzen kommt - die aber nicht in den Zusammenhang der bisher geschilderten Abtragungsvorgänge gehören - ist allein auf die unsachgemäße Pflege, hauptsächlich aber auf die aufgelassenen Kulturen zurückzuführen. Diese Wüstungen sind als Folgen der seit ca. 1952 andauernden Landflucht anzusehen. Sie bilden eines der Kennzeichen der heutigen ligurischen Agrarlandschaft. Die unterschiedlichen Abtragungsverhältnisse auf der N- und S-Seite geben auch eine Antwort darauf, warum in Ligurien überhaupt Terrassen angelegt werden konnten und im emilianischen Apennin nicht. -

Frane sind nicht nur auf das offene Land beschränkt, sondern ereignen sich ebenso im Waldland. Z. B. kann die im März 1961 in 1100 m Höhe am Mte. Penice (71, II NO) erfolgte Frana angeführt werden, wobei ungefähr $1800 \mathrm{~m}^{2}$ Wald auf den Rücken des von Tonen unterlagerten Alberese abglitten (Hangneigung $15 \mathrm{Grad}$ ). Andere derartige Beispiele finden sich bei Trabucco und Almagia, die nachweislich keine Bergstürze gewesen sind und nicht durch menschliche Eingriffe hervorgerufen wurden.

Damit erhebt sich die Frage, ob die Entwaldung - womit der gesamte Wasserhaushalt zusammenhängt - einen maßgeblichen Einfluß auf die Entstehung der Frane ausübt oder gar die alleinige Ursache abgibt.

Nach den Angaben von De Bartolomeis (1840) und C. Rognoni (1897) verminderte sich durch die Rodungsvorgänge im Nordapennin im Verlauf des 18. Jahrhunderts die Quellschüttung in den Sommermonaten auf etwa die Hälfte, während ein erhöhter

6 Es soll betont werden, daß die Menge und Verteilung der Niederschläge auf der ligurischen und emilianischen Abdachung zwar differiert, jedoch keineswegs krasse Unterschiede zeigt. D.h. daß auf keinen Fall allein die hygrischen Verhältnisse für das Auftreten der Frane verantwortlich gemacht werden können. 


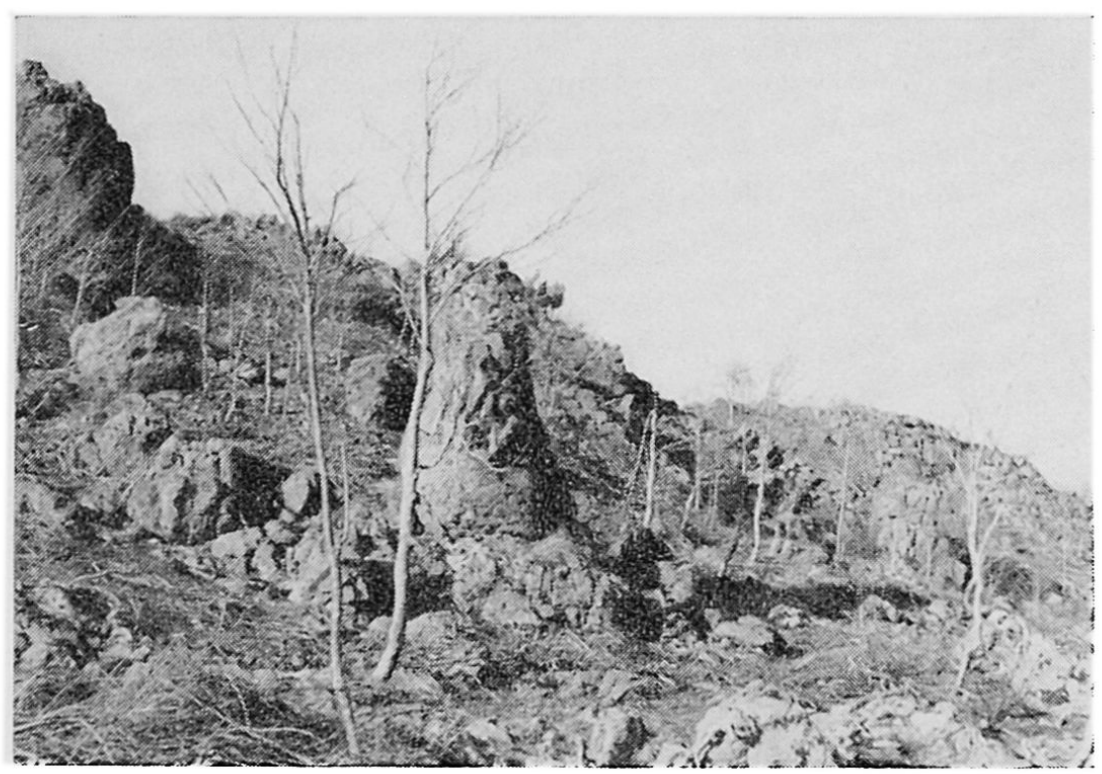

Abb.: 6

Felsruinen und

Blockstreu

Abfluß in den Herbst- und Frühjahrsmonaten eintrat. Parallel dazu setzte eine verstärkte Aufschotterung und eine gesteigerte Lateralerosion in den Mittel- und Unterläufen der Flüsse ein. Die Trebbia erhöhte innerhalb von 100 Jahren ihr Bett bei Bobbio um ca. $2 \mathrm{~m}$. Gleichzeitig verstärkte sich die Tiefeneorsion in den Oberläufen und in den mehr oder weniger periodisch wasserführenden, gefällreichen Torrenten. Auf der topographischen Karte des Herzogtums Parma-Piacenza von 1821/28 (M 1: 86400 ) ist zu erkennen, daß die beiden seitlich die Frana (Beispiel 2, Perino, vergl. S. 232) begrenzenden Bäche schon vor der Entwaldung des flachen Spornes angelegt waren, sich jedoch nur wenig eingekerbt hatten. Vermutlich erfolgte die rasche Eintiefung erst, als die Rodung des Rückens und der tiefer gelegenen Teile am Mte. Poggio Alto einsetzte (72, III NO), (Gründung der Weiler Cognazzo, Pollegri und Columbaro zwischen 1820 und 1840, vordem handelte es sich um 5 einzeln stehende Gehöfte).

Somit ergibt sich, daß Tiefen- und Lateralerosion, die selbstverständlich schon seit dem Aufsteigen des Gebirges wirksam waren, Verhältnisse schufen, die die Entstehung von Franen begünstigten. Aber erst mit dem Beginn der Rodung, die das Wechselspiel von Mikroklima, Wasserhaushalt, Erosion und Verwitterung gründlich veränderte, traten große Rutschungen in erhöhtem Maße auf.

- Daß sich schon kurz nach der Faltung eines aus weichen tonigen Gesteinen bestehenden Schichtverbandes große Rutschungen ereignen können, ist zwar nicht im Untersuchungsgebiet beobachtet worden, aber in Kreta nachzuweisen ${ }^{7}$. G. J. Boekschoten fand $1962^{8}$ auf miozänen Tonen, die mit ca. $8 \mathrm{Grad}$ einfallen, in der Nähe von Iraklion wirr durcheinander liegende Kalk- und Sandsteinmassen, die von jungpleistozänen und rezenten Schuttmassen diskordant überdeckt werden. Der Gleitvorgang dürfte nach Abschluß der letzten reliefbildenden tektonischen Bewegungen auf Mittelkreta stattgefunden haben und stellt nichts anderes als eine fossile Frana dar.

In diesem Zusammenhang sei darauf hingewiesen, daß auch Gleitphänomene einer ganz anderen Größenordnung bis zu einem gewissen Grade mit den Franen verglichen werden können. Die ältere Anschauung des Deckenbaus im Apennin mit den in der Tyrrhenis liegenden Wurzelzonen ist durch die von Migliorini 1933, Anelli 1938, Merla 1952 und M. Richter 1963 gegebenen Deutungen des apenninischen Baustils abgelöst worden. Bei dieser Hypothese der «frane orogeniche» wird ein gravitatives Ab-

7 Synsedimentäre Rutschungen kommen selbstverständlich gleichfalls vor, sollen doch hierbei außer Betracht bleiben.

8 Vortrag in Freiburg 1962 (nicht veröffentlicht). 
gleiten großer Schichtpakete und Eruptivmassen (Ophiolithe) angenommen. Man könnte solche viele km weit erfolgten Gleitvorgänge als Frane im Großstil auffassen. -

\section{BERG- ODER FELSSTÜRZE}

Bergstürze, die ebenso wie die Frane eine Abrißnische und Gleitbahnränder hinterlassen, erfolgen bei steilem Relief, wie es in der Gipfelregion und auf der S-Abdachung vorherrscht. Wäre kein steiles Relief vorhanden, dann käme es in der Mehrzahl aller Fälle zu Franeerscheinungen. Das abgestürzte Material lagert sich kaum jemals in fest umgrenzten Formen ab. Als Ursachen sind die gleichen Kräfte und Vorgänge zu nennen, die schon bei den Franen angeführt wurden; jedoch ist das Einfallen der Gesteinsschichten nicht immer von entscheidender Bedeutung.

Aber auch bei flachen Relief können die bis zu $80 \mathrm{~m}$ hoch über ihre Umgebung herausragenden ophiolithischen Gesteine, die wurzellos in den «Schuppentonen schwimmen», als Bergstürze abbrechen. So wurde der mehr als $70 \mathrm{~m}$ hohe und $100 \mathrm{~m}$ breite Diabasklotz, nahe des Passo del Brallo (71, II SO, westl.von Bobbio), von den in den Tonen weit zurückgreifenden Quellästen des Rio Avagnone unterhöhlt. Die Folge war ein großer Bergsturz. Der Gesteinsklotz löste sich in bis zu $10 \mathrm{~m}^{3}$ große Blöcke auf, die den S-exponierten Hang bei Casa del Campo bedecken. Heute noch erfolgen innerhalb der flach geneigten Sturzbahn $(12 \mathrm{Grad})$, die aber nicht mit der Schichtfläche der ca. 15 Grad einfallenden Tone identisch ist, kleine oberflächliche Rutschungen.

Auf ein ähnliches Ereignis deuten verstreute Ophiolithblöcke unterhalb des Passo di Penice (1143 m, westl. von Bobbio), im Quelltrichter der Anzola oberhalb Selvola (84, IV SO; nahe des Mte. Maggiorasca) und bei Berceto am Cisa-Paß hin (84, II NE). Sehr wahrscheinlich zerstörten die Blöcke bei dem letztgenannten Ort die von dem Langobardenkönig Luitprand (712-744) gegründete Benediktinerabtei. Die morphologischen Verhältnisse lassen diese Schlußfolgerung zu. Ebenso kann es im Zusammenhang mit der Rachelbildung innerhalb der Schuppenton-Serie zum Abbruch großer Kalkbänke kommen, weil die Calanche immer von den Talsohlen her die Bergrücken zerschneiden, so daß die oberhalb davon anstehenden Schichten ihren Halt verlieren und in rascher Bewegung abgleiten können. Anfang der 30-iger Jahre brachen mächtige Kalkschichten der Schuppenton-Serie, auf deren Rücken ca. $1 / 2$ ha Wald stand, in dem nach W-exponierten Tal bei Canossa ab (86, IV NO). Danach konnten die Calanche bis auf die Kammstraße zurückgreifen.

Die drei bisher erwähnten Abtragungsvorgänge können nach ihrem Erscheinungsbilde in eine Reihe gestellt werden. Als Zwischenformen (Vorgänge) - in bezug auf Masse und Geschwindigkeit des abgeglittenen oder abgestürzten Materials - wären noch die Murgänge einzuordnen, die aber im ligurisch-emilianischen Apennin keine maßgebliche Rolle spielen.

\section{QUELLKESSEL (BALZE) UND HOHLWEGE}

Die von G. Braun (1907) als pliozäne Quellkessel beschriebenen, in der italienischen Literatur als Balze bezeichneten Hohlformen sind vereinzelt in den pliozänen Schichten des Subapennins ${ }^{9}$ verbreitet. Das Fliozän steht im nordwestlichen Apennin bei Montebello (Carta Geologica d'Italia, Fo. 71, Voghera, 1929, 1:100 000) an und ist östlich des Chero-Tales (Carta Geologica d'Italia, Fo. 72, Fiorenzuola d'Arda, 1930) als ein breites Band von 4 bis $5 \mathrm{~km}$ entwickelt.

Der größte Quellkessel findet sich bei Lugnano an der linken Seite der Arda (72, II NO und NE). Ein in 4 Teile aufgelöster, bis zu $120 \mathrm{~m}$ tiefer Kessel hat sich in die SEFlanke des Mte. Giogo (460 m) eingefressen. Seine glatten steilen Rückwände weisen

9 Darunter versteht man in der ital. Lit. den nördlichen, emilianischen Apenninrand bis zur Südgrenze des Pliozäns. 


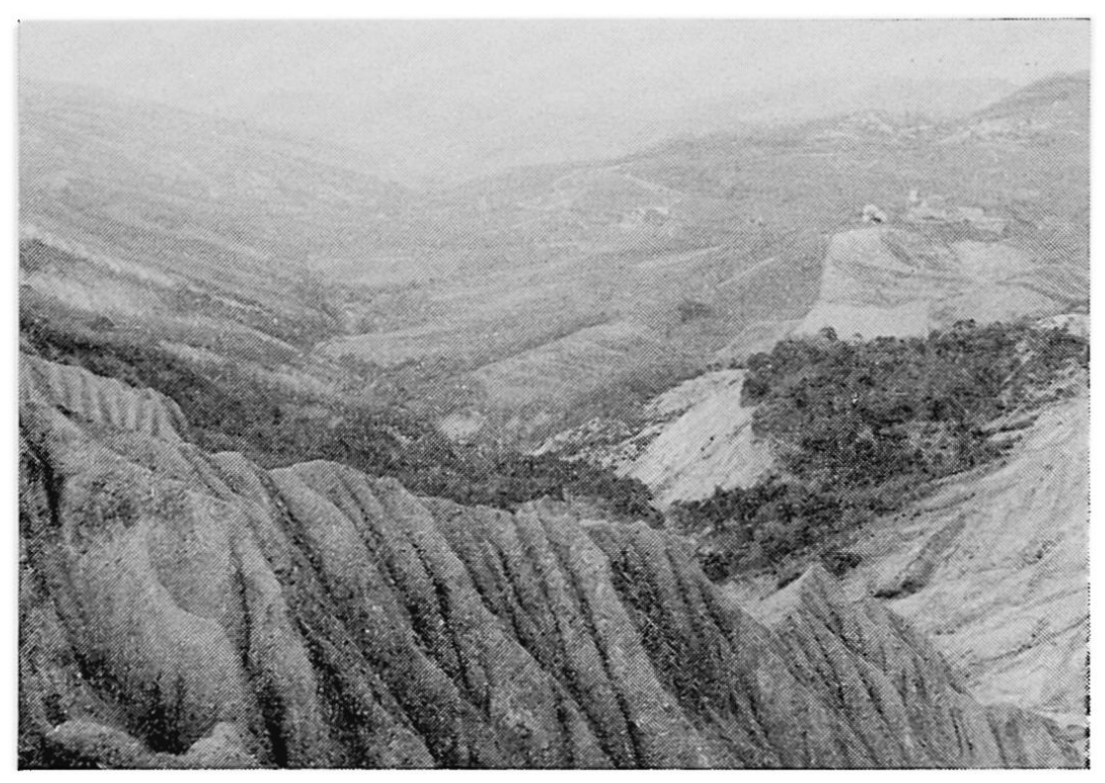

Abb. 5:

Calanche oder Racheln

Winkel von 45 bis 50 Grad auf. Der leicht verfestigte, hellgelbe Sand (Astiano) wird am Grunde der Balze von grauen, tonreichen Mergeln (Piacenziano) unterlagert, die mit ca. 8 Grad nach N einfallen und einen Quellhorizont bilden. Ständig rieselt der Sand von den Wänden und während der Regenperiode brechen große Sandpartien ab. So wird der Kessel jährlich um einige $m$ erweitert, wobei sich durch die verstärkte Quellschüttung im Frühjahr und Herbst die Erosionsleistung bedeutend erhöht. Das Material wird durch den im Kessel entspringenden Bach wegtransportiert. Ein Vergleich mit der bekannten Blaze von San Giusto (Braun 1905) liegt nahe. Eine kleinere, ca. $60 \mathrm{~m}$ im Durchmesser große Balze findet sich nordöstlich von Lugnano.

Andere Hohlformen treten in den Riedeln beiderseits der nach NE zu geöffneten emilianischen Täler auf; es sind Hohlwege. Die Riedel sind aus stark zersetzten pleistozänen Schottern aufgebaut. Neigt der darüber liegende Ferretto zu oberflächlichen Rutschungen (vergl. S. 000), so bilden sich dort, wo der Mensch im Laufe der Jahrhunderte diagonal oder senkrecht die Hügel schneidende Wege anlegte, 3 bis $4 \mathrm{~m}$ tiefe Schluchten mit Steilwänden von 40 bis 50 Grad Neigung. In der Regenzeit verwandeln sich die bis zu $2 \mathrm{~m}$ breiten Sohlen in eine zähflüssige Masse, die im Sommer zu einer harten Kruste erstarrt. Das beste Beispiel ist bei Rivergaro an der Trebbia (72, IV SE) zu sehen.

Während die Quellkessel nur im offenen Land in Erscheinung treten und als Folgen des zerstörten Waldkleides aufzufassen sind, entstehen die Hohlwege durch die Tätigkeit des Menschen (Wagenspuren) auch in bewaldeten Gebieten. Beide Hohlformen kommen nur dort vor, wo die erwähnten Gesteinsverhältnisse gegeben sind (leicht verkittete Sande über Tonen $=$ Quellkessel ; mürbe Schotter $=$ Hohlwege) ; diese sind nur im Subapennin vorhanden. Die klimatischen Verhältnisse sind insofern von untergeordneter Bedeutung, als analog zu den Hohlwegen die Lößschluchten, die hin und wieder im tiefgründig zersetzten Gneis des Südschwarzwaldes eingeschnittenen Hohlwege und die bei Wilhelmy (1958) beschriebenen Maultierhohlwege im zermürbten Granodiorit Kolumbiens in 2300/2500 m Höhe angeführt werden können. Ähnlich verhält es sich mit den Quellkesseln. Auch sie dürfen im wesentlichen unabhängig von klimatischen Einflüssen betrachtet werden; denn es gibt Quellkessel im weichen Kreidesandstein des Daubaerlandes in Nordböhmen, die zwar nicht diese Ausmaße erreichen wie im Nordapennin, doch in die gleiche Kategorie gehören. 


\section{RAC HELN}

Racheln oder Calanche, die dem Landschaftsbild des emilianischen wie auch des ligurischen Apennins bis zu 1300 m Höhe entscheidende Züge aufprägen, sind überall in den harten Gesteinsschichten der Argille scagliose, im Macigno und Alberese anzutreffen. Ihre Form wird durch die im Abstand von 5 bis $10 \mathrm{~m}$ in der Fallinie angeordneten, tief eingeschnittenen Kerben charakterisiert. Dadurch werden die Hänge, Hügel und flachen Rücken in zahllose, fächerförmige, 30 bis 50 Grad steile Firste zerlegt, die selbst wieder von schmalen Runsen zerschnitten werden (Abb. 5).

Die Entstehung hängt aufs engste mit dem Vorgang der Entwaldung zusammen. Die von der Vegetation entblößte Bodenkrume ist in der Regenzeit den intensiven Niederschlägen preisgegeben und wird rasch abgespült. Das auf den oberen Hangpartien flächenhaft abfließende Wasser sammelt sich hangabwärts zu immer stärkeren linearen Wasserfäden, die sich demzufolge zuerst in den unteren Hangabschnitten einschneiden ${ }^{10}$. Die entstandenen Kerben vergrößern sich im Laufe weniger Jahre verhältnismäßig schnell, und zugleich setzt an den stehengebliebenen Graten eine weitere Zerschneidung ein. Das abgespülte Material wird von den Torrenten wegtransportiert, so daß sich keine Ablagerungsformen bilden können.

$\mathrm{Da}$ die Rachelbildung eine Folge der Zerstörung des natürlichen Vegetationskleides darstellt, kann westlich von Bobbio (71, II NE) deutlich beobachtet werden.

In den 30-iger Jahren wurde ein Teil des $\mathrm{N}$-exponierten, ca. $35 \mathrm{Grad}$ steilen Hanges an der Costa Ferraia (1029 m), der in ein Gewirr von Racheln aufgelöst war, vom Gipfel bis zum Torrentenbett der Bobbio $(350 \mathrm{~m}$ ) hinab aufgeforstet. Heute sind die ehemaligen Calanche von einer bis $\mathrm{zu} 20 \mathrm{~cm}$ mächtigen Bodenschicht bedeckt und so stark verwischt, daß nur noch ein unruhiges, aber keineswegs etwa scharf modelliertes Relief vorhanden ist. Die Abspülungsvorgänge sind vollständig zum Stillstand gekommen. Demgegenüber stellt der weiter östlich anschließende, kahle Hang eine typische durch Racheln zerrissene Bergflanke dar. Daß dies kein Einzelfall ist, zeigt der mit Kiefern aufgeforstete Hang (Costa Serba), $350 \mathrm{~m})^{11}$ oberhalb von Sestri Levante auf der ligurischen Abdachung.

Die Racheln dürfen, was Aussehen und Entstehungsursachen anbelangt, am ehesten mit den Steppenschluchten (owrags) Südrußlands und den «gullies» der USA (eine Art soil erosion) gleichgesetzt werden. Wichtig ist, daß sie ihre prägnanteste Ausgestaltung dann erfahren, wenn in den Jahresgang eine sommerliche Trockenzeit eingeschaltet ist und gelegentlich intensive Gewittergüsse niedergehen. Das ist in den nördlichsten Teilen des emilianischen Apennins ${ }^{12}$ und vielfach in den vergleichsweise genannten Gebieten der Fall ${ }^{13}$.

\section{BLOCKMEERE, BLOCK- UND SCHUTTHALDEN}

Die Hänge der Gipfelregion sind in der Umgebung des Mte. Maggiorasca (1803 m) und Mte. Nero (1753 m), (84, IV SO), wo ophiolithische Gesteine (Serpentinit, Pillowlaven, Spilitbrekzie) anstehen, auf einer Fläche von $1,5 \mathrm{~km}^{2}$ mit dichter Blockstreu bedeckt. Dazwischen finden sich im Abstand von 40 bis $50 \mathrm{~m}$ zahlreiche, 3 bis $5 \mathrm{~m}$ hohe Felsstutzen (-ruinen) (Abb. 6), von denen aus - je nach dem Böschungswinkel - 200 bis $3000 \mathrm{~m}^{2}$ große Blockmeere oder Blockhalden hangabwärts ziehen. Das größte Blockmeer überdeckt die Kuppe (10 bis 12 Grad Neigung) des Tana di Nero (1680 m).

Die bis zu 1,5 m großen Serpentinitblöcke sind scharfkantig, die bis $\mathrm{zu} 70 \mathrm{~cm}$ im Durchmesser messenden Pillowlavaköpfe gemäß ihrer kugeligen Absonderung rund. Nur der SE-Hang des Mte. Nero ist mit scharfkantigem Serpentinschutt überzogen,

10 Demnach sollte eine Aufforstung immer vom Gipfel zum Bergfuß hin erfolgen.

11 94, NE.

12 Das drückt sich in den Mittelwerten aber nicht aus!

13 Weniger gut und zahlreich ausgebildete Racheln gibt es in ganz Mitteleuropa. 


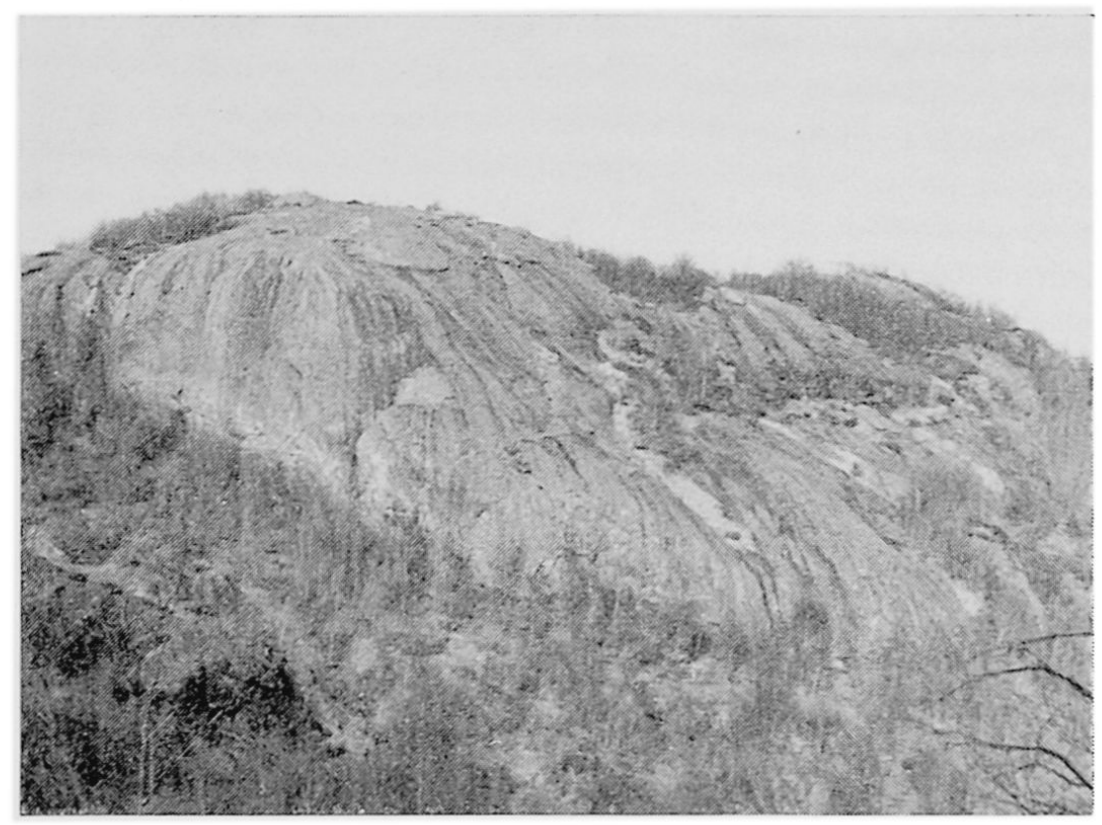

Abb. 7:

* Glockenberg »

der eine $200 \mathrm{~m}$ breite und $30 \mathrm{Grad}$ steile Halde bildet. Die Block- und Schuttbildung ist durch das z. T. eng-, z. T. weitständige Kluftsystem der harten Ophiolithe zu erklären und bedarf wohl keiner weiteren Diskussion.

Die Felsstutzen, die als Trümmerquellen in Frage kommen, können als verstürzte Felsburgen angesprochen werden. Form und Lagerung der unterhalb davon liegenden Blöcke lassen erkennen, wie sie ursprünglich aufgebaut waren, d. h. als Felsburgen aus anstehendem Gestein. So ist das Blockmeer am Tana di Nero aus einer SerpentinitFelsburg hervorgegangen, was sich aus der Mächtigkeit der Blockpackung auf der Kuppe ableiten läßt. Weiter abwärts sind die Blöcke viel weniger dicht übereinander getürmt. Rutschbewegungen der Blöcke über kurze Entfernungen und vereinzelte Blockbildung in situ sind jedoch nicht ausgeschlossen. Diese Vorgänge sind am Aufbau des Blockmeeres beteiligt gewesen, nicht aber als deren alleinige Ursache aufzufassen.

Zeitlich gesehen, ist die Bildung der groben Trümmeransammlungen wie auch das Herauslösen aus dem Gesteinsverband im Postglazial erfolgt. Das kann einerseits daraus geschlossen werden, daß sie in einer Höhe in Erscheinung treten, wo sie - wären sie älter - von den Gletschern der Eiszeit hätten verschleppt werden müssen, andrerseits aus ihrer heutigen lockeren Lagerung. Letztere ist nicht damit zu erklären, daß auf den verhältnismäßig flach geneigten Hängen innerhalb der Blockmeere eine Ausschwemmung des Lockermaterials vorsichgeht. Das Gegenteil ist der Fall. Überall finden sich in den Zwischenräumen der Blöcke abgeplatzte Gesteinsbruchstücke und feinerdiges Material, in dem sich hin und wieder einige (verkrüppelte) Buchen angesiedelt haben. Die in situ-Verwitterung ist in vollem Gange und die Vegetation versucht langsam vorzudringen. Die Block- und Schutthalden in dieser Höhe (über $1650 \mathrm{~m}$ ) werden ständig weitergebildet, ohne daß die Vegetation in den locker aufgetürmten Blöcken oder in dem scharfkantigen Schutt Fuß zu fassen vermag.

Anders verhält es sich in den tieferen Lagen, so z. B. im Gebiet des unteroligozänen Konglomerats zwischen Scrivia und dem Oberlauf des Scuropasso (71, alle Blätter von III und IV sowie von 83 alle Blätter von IV). Das Gestein hebt sich von dem südöstlich davon beginnenden Alberese deutlich ab und bildet nach SE zu eine Steilstufe (entgegengesetzt dem Einfallen). In engen Schluchten wird es von der Vobbia und Borbera durchflossen. Das fest verbackene Gestein zerfällt nicht in seine Gesteinskomponenten, sondern bricht in großen Blöcken, manchmal auch wie bei Pietrabissara (Scrivia) schalenförmig ab. Sein südlichstes Vorkommen liegt am Mte. Maggio (978 m), 
(83, IV SO), dessen östlicher Steilabfall mit Riesenblöcken vom Ausmaß von Zehnern von Kubikmetern übersät ist. Sie bilden in ca. $800 \mathrm{~m}$ Höhe eine Blockhalde. Die Zwischenräume der Felstrümmer sind vollständig mit kleinstückigem Schutt und Erdreich ausgefüllt und mit Eichenbüschen bestanden. Nur selten sind frische Bruchflächen an den Blöcken festzustellen. Vereinzelte Blöcke des Konglomerats liegen noch weiter südlich auf den Hängen zwischen der Scrivia und Brevenna. Ihre Herkunft ist aus den heutigen morpholoigschen Verhältnissen nicht zu erklären. Sehr wahrscheinlich brachen sie zu einer Zeit ab, als das Gestein noch weiter nach $S$ zu verbreitet war. Das dürfte während des Pleistozäns der Fall gewesen sein; denn zu dieser Zeit lagen die Hänge im periglazialen Klimabereich, wo eine große Verwitterungsaktivität gegeben war. Auch für die Entstehung der erwähnten Blockhalde und aller anderen groben Trümmeransammlungen unterhalb von $1650 \mathrm{~m}$ - die zwar in geringem Maße noch weitergebildet werden - ist diese zeitliche Einstufung anzunehmen.

Demgegenüber sind die überall im ligurisch-emilianischen Apennin unterhalb von $1650 \mathrm{~m}$ liegenden Schutthalden (z. B. Mte. Barbarino, $405 \mathrm{~m}$, Ophiolith bei Bobbio) mit allen Merkmalen aktiver Weiterbildung behaftet, was hauptsächlich auf das engmaschige Kluftnetz der Trümmerquellen zurückzuführen ist. Frost und Insolation bewirken in allen Höhenstufen das Absprengen von Gesteinspartikeln, wenn auch mit unterschiedlicher Intensität.

\section{BESONDERE VERWITTERUNGSFORMEN}

Vielfach bilden verstürzte Felsburgen die Gipfel (Mte. Nero) oder ragen, eigentümlich geformt, aus den Hängen heraus. Die Pillowlavablöcke türmen sich bis zu $10 \mathrm{~m}$ Höhe auf, während die harte feinbrockige Spilitbrekzie in $1490 \mathrm{~m}$ Höhe am SE-Hang des Tana di Nero mehrere nebeneinander stehende «Riesenkartoffeln» von $15 \mathrm{~m}$ Höhe mit ziemlich glatter Oberfläche bildet. Eine glockenbergähnliche Form zeigt die kahle Kuppe des Pietra Spaccata (1404 m; in der Nähe des Mte. Maggiorasca), die aus grober Spilitbrekzie aufgebaut wird und die Umgebung um $30 \mathrm{~m}$ überragt (Abb. 7). Der konvexen Krümmung angeglichen, bedecken bis zu $10 \mathrm{~m}^{2}$ große und 30 bis $40 \mathrm{~cm}$ dicke Verwitterungsschuppen den Gipfel. Am Bergfuß sind überhängende Steilabbrüche festzustellen, die in ihrem Ausmaß der oberflächenparallel verlaufenden Klüftung entsprechen. Die in der Fallinie nebeneinander angeordneten schwarzen und hellgrauen Streifen (im Bild sichtbar), sind mit Flechten überzogene Rillen. Sie sind gemäß den herausgewitterten Gesteinsbrocken rauh. Unterhalb des «Glockenberges» liegen einzelne Schuppen, die zu Grus verwittern.

Diese besonderen Verwitterungsformen, die alle im ehemals vergletscherten Gebiet vorkommen, weisen keine Spuren eiszeitlicher Überformung (Gletscherschliffe) auf. Ihrer geringen Höhe wegen ist auch nicht anzunehmen, daß sie über das Eis hinausgeragt haben. Sie müssen - wie die hier vorkommenden Trümmeransammlungen - gleichfalls nacheiszeitlicher Entstehung sein und ihre Formen sind durch das Kluftsystem bestimmt. Die «Riesenkartoffeln» entsprechen dem weitmaschigen Kluftnetz, das teils durch die Tektonik bedingt (Transport der Ophiolithe während des gravitativen Abgleitens), teils aber auch primärer Entstehung sein kann, da es sich um eine Eruptivbrekzie handelt. Davon zu unterscheiden sind diejenigen Klüfte, die bis zu einem gewissen Grade das Relief der Erdoberfläche nachzeichnen. Ihr Abstand wird mit zunehmender Tiefe größer und ausgeglichener; sie erklären sich aus den Einwirkungen der jahres- und tageszeitlichen Temperaturschwankungen im Zusammenhang mit dem Sickerwasser auf das Gestein. Der Vorgang und das Erscheinungsbild werden Bathroklase genannt ${ }^{14}$. Die Desquamation des aus grober Spilitbrekzie aufgebauten Glockenberges darf durch diesen Prozeß, der zweifellos auch in der Gegenwart wirksam ist,

14 E. SueB, Mitt. Geol. Ges. Wien. VI. Bd. 1913 und Verf. 1959. 


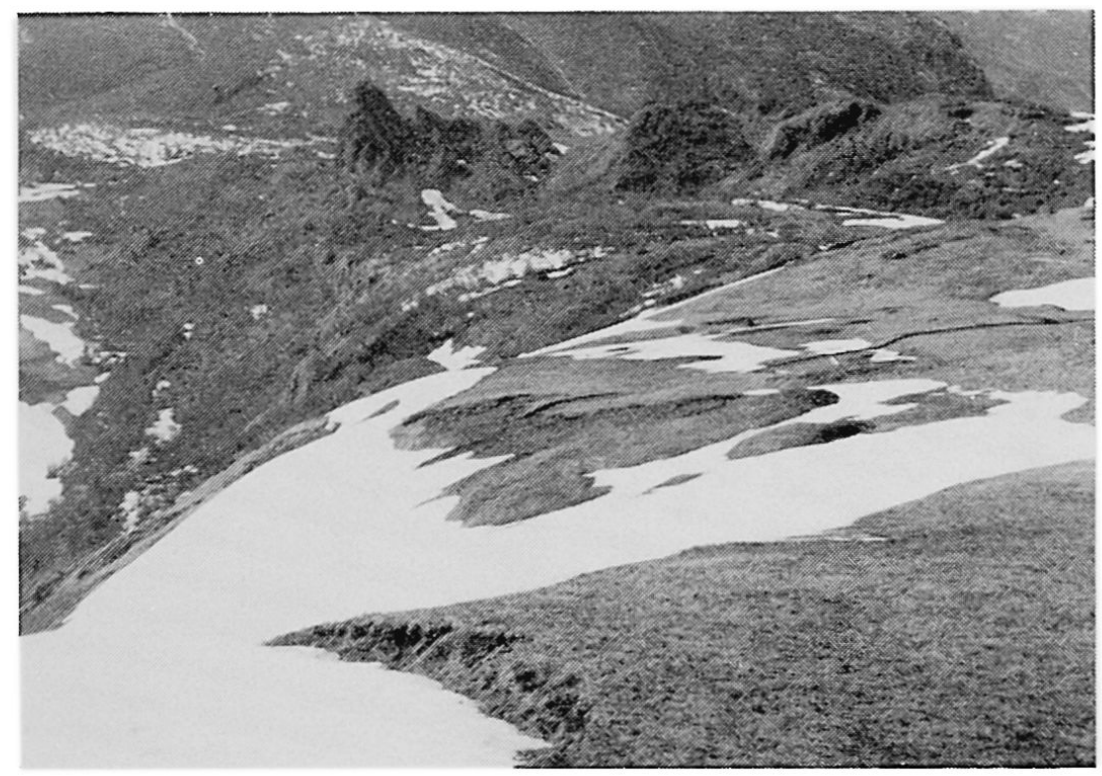

Abb. 8:

Rasenschälen in $1650 \mathrm{~m}$ Höhe

erklärt werden. Die Klüfte können nicht primärer Entstehung sein, da die Verkittung der verschiedenen Gesteinskomponenten dieser Brekzie sedimentär erfolgte ${ }^{15}$. Eine Druckentlastung kann hier ausgeschlossen werden.

\section{REZENTE SOLIFLUKTIONSERSCHEINUNGEN}

Auf der schmalen, unbewaldeten Einsattelung zwischen dem Mte. Maggiorasca und Mte. Nero konnten im April 1961 in 1650 m Höhe gebundene Solifluktionserscheinungen, Formen eines sog. Rasenschälens, beobachtet werden (Abb. 8). Die Rasennarbe am Kamm und auf dem ca. 20 Grad steilen NW-Hang war durch eine Vielzahl hufeisenförmiger Abrißnischen von 1 bis $3 \mathrm{~m}$ Durchmesser aufgerissen. Am Hang hing die Rasendecke über dem Abrißgebiet, das noch mit Schneeflecken ausgefüllt war. Die losgerissene Rasendecke bildete am schneefreien Hang bis zu $50 \mathrm{~cm}$ hohe, parallel zu den Isohypsen verlaufende Wülste. Diese Erscheinungen, die auf Kammeisbildung und Schneefleckenerosion zurückzuführen sind, konnten ebenso auf den Hängen des Mte. Lesima in $1690 \mathrm{~m}$ Höhe in NE-Exposition festgestellt werden. Sie geben einen Anhaltspunkt dafür, daß diese Höhenstufe des nordwestlichen Apennins zum Bereich des rezenten Periglazialklimas gehört. Damit ist zugleich ausgesagt, da $\beta$ die groben Trümmeransammlungen, die dazugehörigen Felsstutzen, Felswände und besonderen Verwitterungsformen über $1650 \mathrm{~m}$ in den Bereich des heutigen Periglazialklimas hineinragen. Ihre Weiterbildung wird durch die hier intensiv wirkenden physikalischen Agenzien erklärlich.

Demgegenüber sind Polygon- und Steinstreifenböden nirgends beobachtet worden. Das mag daran liegen, daß die flachen, schmalen Rücken sowie die entwaldeten Hänge der Beweidung unterliegen, so daß eventuell gebildete Strukturböden schon im Anfangsstadium zerstört werden.

Für die Erhaltung von fossilen Periglazialerscheinungen ist der ligurisch-emilianische Apennin schlecht geeignet; denn nach den bisher geschilderten klimatischen, morphologischen Bedingungen und anthropogenen Einflüssen sind fast alle Hänge einer mehr oder weniger intensiven rezenten Abtragung ausgesetzt. Aber schon aus dem gesteinsmäßigen Aufbau, z. B. der weiten Verbreitung der Tone, ist zu schließen, daß die Solifluktionsvorgänge während der Eiszeit - zumindest während der Maximalvergletscherung

15 Analyse von H. D. Trochim, Mineralogisches Inst. Freiburg/Brsg. 
- die gesamte N-Abdachung bis zur Poebene hinab erfaßten. Diese Vermutung dürfte insofern gerechtfertigt sein, als zu berücksichtigen ist, daß der kontinental-klimatische Charakter (Temperatur!) der Apenninnordseite ungefähr dem des Alpenrandes westlich Turin und Ivrea entspricht. Dort konnten in den Moränenamphitheatern der Dora Baltea und Dora Riparia u. a. Fließerdehorizonte in 250/300 m Höhe nachgewiesen werden (Fränzle 1959). Auf der maritim beeinflußten S-Abdachung dagegen, die ähnliche klimatische Züge wie Korsika trägt, dürfte die untere pleistozäne Solifluktionsgrenze nicht tiefer als $500 \mathrm{~m}$ gelegen haben.

W. Klaer diskutierte 1956 das Problem der Schnee- und Solifluktionsgrenzen für den Raum zwischen Alpen und Atlas. Dabei ergab sich, daß die eiszeitliche Schnee- und die rezente Solifluktionsgrenze von $N$ nach $S$ zu aneinanderrücken und sich bei ca. $40 \mathrm{Grad}$ n. Br. kreuzen. Nimmt man an, daß die pleistozäne Schneegrenze im nördlichen Apennin bei $1400 \mathrm{~m}$ gelegen hat (v. Klebelsberg 1948), die heutige Solifluktionsgrenze bis $1650 / 1700 \mathrm{~m}$ hinabreicht, so beträgt der Abstand im emilianisch-ligurischen Apennin (441/2 Grad) 250 bis $300 \mathrm{~m}$ (Alpen $800 \mathrm{~m}$, Korsika $200 \mathrm{~m}$ ).

v. Klebelsberg setzte aber westlich des Giovi-Passes im Ligurischen Massiv (Gruppo di Voltri) die eiszeitliche Schneegrenze unter $1200 \mathrm{~m}$ an. Er bezieht sich dabei auf ein von F. Sacco (1937) auf der N-Seite des Mte. Beigua $(1287 \mathrm{~m})$ festgestelltes Kar (Karboden bei $1220 \mathrm{~m}$ ) und stützt sich im übrigen auf die Annahme, daß das Ligurische Massiv den niederschlagsreichsten Teil des gesamten Gebirges bilde. Gegen beide Beweisargumente sind Einwände zu erheben. Zum einen werden die Niederschlagsmengen am Mte. Beigua von denen des Mte. Maggiorasca um nicht weniger als $900 \mathrm{~mm}$ übertroffen, zum anderen stellt das «Kar» eine Mulde ohne eigentlichen Boden mit flachen «Rück- und Seitenwänden» dar. Vergleicht man damit die gut ausgebildeten Kare in der Hochregion des ligurisch-emilianischen Apennins, z. B. Mte. Aiona (Karbodenhöhe bei $1320 \mathrm{~m}$ ), so dürfte es sich am Mte. Beigua um nichts anderes als eine Firnmulde handeln.

Daraus kann gefolgert werden, daß die eiszeitliche Schneegrenze im Untersuchungsgebiet tiefer als $1400 \mathrm{~m}$, im ligurischen Massiv ohne Frage nicht unter $1200 \mathrm{~m}$ gelegen haben muß. Damit vergrößert sich der Abstand zwischen eiszeitlicher Schneegrenze und rezenter Solifluktionsuntergrenze um ca. $100 \mathrm{~m}$, so daß die beiden Grenzlinien - von $\mathrm{N}$ nach S - auf 350 bzw. $400 \mathrm{~m}$ auseinanderrücken. Dieser Wert fügt sich gut in das von Klaer aufgestellte Schema ein. Eine Korrektur ist aber auf der N-Seite des Apennins vorzunehmen, wo die rezente Solifluktionsuntergrenze ungefähr der des Alpennordrandes vergleichbar ist ${ }^{16}$. Der Nordapennin stellte also im Pleistozän - wie auch in der Gegenwart - eine Klimascheide ersten Ranges dar.

In Vorarlberg tauchen gebundene Solifluktionsvorgänge in ca. $1600 \mathrm{~m}$ auf.

\section{SCHLUSSBETRACHTUNG}

Faßt man die an einzelnen Beispielen aufgezeigten Beobachtungen zusammen, so ergibt sich, daß für die Abtragungsvorgänge im ligurisch-emilianischen Apennin die durch die Tektonik geschaffenen Großformen von ganz entscheidender Bedeutung sind. Der Abbruch zum Ligurischen Meer verlieh der S-Seite des Gebirges ein steiles Relief. Die kurzen Bäche zerschnitten die ligurische Abdachung intensiv, doch die Abtragungsvorgänge in ihrer Gesamtheit vermochten nicht, die herauspräparierten Sporne einzuebnen. Denn sollte die Abtragung der Ausbildung einer tiefgelegenen Fläche zustreben, so dürfte die Kürze der Zeit seit dem Tertiär nicht ausgereicht haben; überdies wirkte dem die Hebung des Gebirges, die bis zur Gegenwart anhält, entgegen.

Im Vergleich dazu weist die N-Seite noch zahlreiche Verebnungsflächen und breite Rücken auf, die von den wenig gefällreichen, jahreszeitlich sehr unterschiedlich wasser-

16 In Vorarlberg tauchten gebundene Solifluktionsvorgänge in ca. $1600 \mathrm{~m}$ auf. 
führenden Flüssen und Bächen relativ wenig zerschnitten, geschweige denn aufgezehrt werden konnten.

Die klimatischen Verhältnisse, insbesondere Menge und Verteilung der Niederschläge, konnten die durch tektonische Vorgänge geschaffenen Großformen ${ }^{17}$ nicht wesentlich verändern. Sie modifizierten nur geringfügig die Abtragung.

Als anthropogen bedingte Formen und Vorgänge, die allein auf die Entwaldung und ihre Folgen zurückzuführen sind, dürfen 1. die Erdschlipfe genannt werden. Sie ereignen sich im offenen Land, wo anlehmiger Detritus, lehmige Böden oder tonige Schichten vorkommen. Nur selten treten sie auf der terrassierten S-Abdachung, häufig dagegen auf der emilianischen Seite in der Schuppenton-Serie auf. Ihre obere Grenze liegt bei ca. 1200 m. 2. Quellkessel und Hohlwege sind auf den schmalen Saum des Subapennins beschränkt. Erstere sind an die pliozänen Sande mit darunter lagernden Tonen gebunden, letztere an die stark zersetzten pleistozänen Schotter, die die Riedel beiderseits der in die Poebene mündenden Täler aufbauen. 3. Racheln oder Calanche sind im gesamten ligurisch-emilianischen Apennin bis in eine Höhe von ca. $1300 \mathrm{~m}$ anzutreffen, erfahren aber ihre beste Ausbildung am relativ sommertrockenen Rand des emilianischen Apennins in den harten Schichten der Argille scagliose-Serie.

Dem stehen 4. Frane und Bergstürze gegenüber, die in vielen Fällen schon seit Beginn der Gebirgsbildung ausgelöst, jedoch durch die Auswirkungen der Rodungen außerordentlich verstärkt worden sind. Daß die Frane hauptsächlich nur im emilianischen Apennin bis zu $1100 \mathrm{~m}$ Höhe verbreitet sind, ist weniger eine Folge der Klima-als der Reliefverhältnisse. Für die Frane bildet die Lagerung der Gesteine - wasserdurchlässige Gesteinsschichten über wasserundurchlässigen, hangparallel einfallenden Tonen - eine wesentliche Voraussetzung, während für die Bergstürze, die überall im ligurisch-emilianischen Apennin erfolgen können, diese Fakten von mehr untergeordneter Bedeutung sind.

Die Ursache für die großen Massenbewegungen bildet die Unterschneidung der Gesteinsschichten durch die Wasserläufe; hinzu kommt als weiteres Moment u. U. der Straßen- und Eisenbahnbau. Als auslösende Ursache kommen im Nordapennin Erdbeben kaum in Frage. Stürzte zwar 1828 der Castellturm von Bagnaria infolge eines Erdbebens ein, so riefen die Erschütterungen aber keine Bergstürze oder Frane hervor.

Alle genannten Abtragungsvorgänge ereignen sich am häufigsten oder werden weiter gebildet in den niederschlagsreichen Herbst- und Frühjahrsmonaten.

Allein durch klimatische Gegebenheiten ist 6. das Rasenschälen in der über $1650 \mathrm{~m}$ hoch liegenden Gipfelregionen zu deuten, die in den periglazialen Klimabereich hineinragt.

7. Blockmeere, Blockhalden und besondere"Verwitterungsformen sind in den harten ophiolithischen Gesteinen und Konglomeraten verbreitet. Ihre Entstehung verdanken sie dem weitmaschigen Kluftnetz und den intensiven Frostsprengungsvorgängen während des Periglazialklimas. Soweit sie in der über $1650 \mathrm{~m}$ liegenden Höhenstufe auftreten, werden sie noch heute weiter gebildet. 8 . Schutthalden, welche da und dort die steilen Flanken der Ophiolithklötze bedecken, kommen von der obersten Höhenstufe bis in Lagen von $400 \mathrm{~m}$ Höhe vor und lassen alle Merkmale rezenter Weiterbildung erkennen. Für sie gilt als Voraussetzung das engständige Kluftnetz der harten Gesteine.

Die Exposition spielt für alle aufgezählten Verwitterungs- und Abtragungsvorgänge - mit Ausnahme der gebundenen Solifluktion (N-Sektor) - keine maßgebliche Rolle.

So erklären sich die unterschiedlichen Vorgänge und Formen auf der S- bzw. N-Seite und in der Gipfelregion aus dem Zusammenwirken der geologisch-physischgeographischen und anthropogenen Einflüsse. Die menschlichen Eingriffe steigerten zwar insgesamt die Abtragung und verursachten das Auftreten von bislang unbekannten Vorgän-

17 Steile S- und flache N-Seite. 
gen, die der Kategorie der «soil erosion» zugerechnet werden können, doch ist die Entstehung der Vorgänge an die geologisch-lithologischen Verhältnisse gebunden. Die klimatischen Verhältnisse sind für die Ausprägung der Formen und die Intensität der Vorgänge wesentlich, doch dürfen sie nicht überbewertet werden.

Weiterhin ist festzustellen, daß im verhältnismäßig niedrigen und schmalen ligurisch-emilianischen Apennin recht verschiedene Klimaregionen, bzw. -gürtel (maritimmediterran, kontinental-randmediterran, «montan», periglazial) auf engerem Raum zusammengedrängt sind, als das in anderen, selbst höheren südeuropäischen Gebirgen der Fall ist.

\section{LITERATUR}

Almagia, R.: Studi geografici sulle frane in Italia. Mem Soc. Geografica Italia, Roma 19o7; - Note ad un abbozzo di carte delle distribuzione delle frane nella penisola italiana. Com. Ital. per lo studio delle calamita. Roma 1931. Braun, G.: Beiträge zur Kenntnis der Morphologie des nördlichen Apennins. Z.G.E. Berlin 1907. De Bartolomeis, G.L.: Notizie topografiche e statistiche sugli Stati Sardi. 4 Bde. Torino 18to/47. Castiglioni, B.: Ricerche morfologiche nei terreni pliocene dell'Italia Centrale. Publ. Istituto Geogr. Uni. di Roma, A 4, 1935. Fischer, Th.: Italien. Landeskunde von Europa, Bd. 3. Herausgeb. von A. Kirchhoff. Leipzig 1893. Fränzle, O.: Untersuchungen über Ablagerungen und Böden im eiszeitlichen Gletschergebiet Norditaliens. Erdkunde XIII, 1959. Kanter, H.: Junge Abtragungserscheinungen in den Tertiärgebieten des östlichen Kalabrien und eines Teiles der südlichen Basilikata. Z. f. Geomorphologie +, 1929. Klaer, W.: Verwitterungsformen im Granit auf Korsika. PM Ergh. 261, 1956. Klebelsberg, R. v.: Handbuch der Gletscherkunde und Glazialgeologie. Bd. II, Wien 1949. Maull, O.: Handbuch der Geomorphologie. 2. Aufl. Wien 1958. Penta, F.: Sulla classifica delle frane interessanti l'ingegnera. La ricerca scientifica. 1959. Reutter, K. J.: Geologische Untersuchungen im Gebiet zwischen Bedonia und Varese Ligure im Nordapennin. Diss. F. U. Berlin 1960. Rognoni, C.: Sull'antica agricoltura parmense. Parma 1897. Roveri, E.: Su alcuni movimento franosi dell'Appennino emiliano. Asfalti, Bitumi, Catrami. Nov./Dic. 1960. Sacco, F.: Il glacialismo nel gruppo del Mte Ajone. Atti Acc. sc. Torino 1937/38. Sames, C. W.: Zur Geologie des Nordapennins im Raum Bedonia. Diss. F. U. Berlin 1960. Tichy, F.: Beobachtungen von Formen und Vorgängen «mediterraner Soliflutkion» Dt. Geographischer Tag 1959. Trabucco, G.: Le frane dell'alto Piacentino. Piacenza 1889. Ullmann, R.: Verwitterungsdecken im Südschwarzwald. Bericht Naturforschende Ges. Freiburg, 5o, 1960. Wilhelmy, H: Klimamorphologie der Massengesteine. Braunschweig 1958. Zaruba, Q. und Mencl, V.: Ingenieurgeologie. Verlag der tschechoslovakischen Akademie der Wissenschaften, Prag. Akademie-Verlag Berlin 1961.

\section{PROCESSES OF WEATHERING AND EROSION IN THE NORTHERN APPENNIN}

The northern Appennin consists of clays, marls, with intercalarated limestone and sandstone layers, ophioliths, and conglomerats. The steep descent towards the Ligurian Sea caused by tectonic movements gave a very marked relief to the southern side, whereas the northern side slopes gently down to the Padania. These differences of relief were not eliminated by climate. Although amount and distribution of rainfall modified the erosion, they must not be overestimated in theireffects. Only human interference with natural vegetation accelerated the erosive processes or started new ones, as e.g. mudflows, «balze», gullies (calanche), and sunken roads. «Frane» (slow moving landslides) and landslides have been caused since the lifting of the mountains. They were extremely intensified by deforestation. Frane are only found on the northern slope, landsides in places with steep relief (especially in Liguria). Frane are confined to places where permeable beds are underlain by impermeable ones. For landslides this is of less importance. The cause of large mass movements is the untercutting of rock-strata by torrents. All processes take place mainly during the rainy winter months. Solifuxion occurs in the periglacial climate above $1650 \mathrm{~m}$. Agglomerations of boulders and other special forms produced by weathering are due to a wide system of jointing in hard rocks and the expansive force of ice in periglacial regions. Screes are found in altitudes where hard rocks with a narrow system of jointing occur. The intensity oft heir development is related to climate conditions. 